\title{
National Dengue Day (India): Combatting the Vector-Borne Public Health Threat
}

Dengue is a mosquito-borne viral infection causing a severe flu-like illness and sometimes causing a potentially lethal complication called severe dengue. ${ }^{1}$ It is caused by the dengue virus (DENV, 1-4 serotypes) and transmitted by bite of infected Aedes aegypti mosquito which bites during daylight hours. National Dengue Day is observed in India on May 16 every year with the recommendation of Ministry of Health and Family Welfare, Government of India to create awareness about dengue; and to intensify preventive measures and preparedness for the control of disease in the country before transmission season starts. ${ }^{2}$

A dramatic rise has been seen in the global incidence of dengue in recent decades. It is a life-threatening infection and results in many deaths each year. About half of the world's population is now at risk. There are an estimated $100-400$ million infections each year. ${ }^{3}$ The first epidemic of clinical dengue-like illness was recorded in Madras in 1780 and the first virologically proved epidemic of dengue fever in India occurred in Calcutta and Eastern Coast of India in 1963-1964. $\cdot^{4^{-6}}$

As per the National Vector Borne Disease Control Programme (NVBDCP), Ministry of Health \& Family Welfare, Government of India, 136422 cases and 132 deaths were reported in India in 2019. ${ }^{7}$

Aedes aegypti mosquito, also known as the yellow fever mosquito, is responsible for spreading dengue fever, yellow fever viruses, Zika fever, chikungunya and Mayaro. ${ }^{8}$ These mosquitoes are found worldwide (except in Antarctica) and can live in a large and eclectic array of ecosystems, from tropical forests to urban areas and tundra. ${ }^{9}$

After an infective bite, the subject develops symptoms in 3-14 days which generally a high fever, headache, muscle pain, bone and joint pain, nausea, vomiting, pain in eyes, rashes and swollen glands. Severe dengue or dengue hemorrhagic fever is a life- threatening condition with severe abdominal pain, persistent vomiting, bleeding gums, bleeding nose, hematuria, hematemesis, melena, bleeding under the skin, difficulty in breathing, fatigue, restlessness and cold-clammy skin. A small proportion of cases may show fatal disease as dengue shock syndrome. Infected patients become a source of infection and can transmit the same to other via Aedes mosquitoes during 4-5 days after onset of symptoms.

Children are also too prone as they have a growing immune system and are not able to fight the disease effectively. Dengue fever not only affects liver and leads to a reduction in platelet count but also affects kidney, lung, heart and central nervous system. Very low levels of platelets can result in internal bleeding and shock, which leads to death from multi-organ failure.

Virological and serological tests can be used for diagnosis of dengue infection. The virological tests directly detect elements of the virus and serological tests detect human-derived immune components that are produced in response to the virus. Patient samples collected during the first week of illness should be tested by both serological and virological methods (RT-PCR). IgM and IgG tests detect dengue in the initial stages of infection, but since they throw up false positive and false negative results, a confirmatory test is needed. A positive IgG but a low or negative IgM indicates past dengue infection. The NS1 Elisa-based antigen confirmatory test is done three days after symptoms appear.

No specific antiviral medication for dengue exists. Appropriate and timely clinical diagnosis by physician followed by proper clinical management helps in reducing the fatality rate. Use of analgesics (paracetamol), taking plenty of fluids and rest are important aspects of treatment. Precautions should be stressed upon and mild dengue might turn into a fatal condition. The common precautionary measures

(c) Mandeep Singh.. This is an open access article distributed under the terms of the Creative Commons Attribution License CC-BY-NC 4.0, which permits unrestricted use, distribution and reproduction in any medium, provided the use is not commercial and the original author(s) and source are cited.

International Healthcare Research Journal 2020;4(2):26-27. 
include covering water containers with lids, use of larvicidal agents in water storage tanks, use of insecticidal sprays in home and surroundings, covering arms and legs during transmission season, using mosquito nets, insecticide treated bed nets, mosquito repellent sprays, creams, lotions, coils and vaporizers to prevent mosquito bites, protecting dengue patient from further mosquito bites to prevent further spread of dengue to other subjects and preventing unnecessary stagnation of water.

The prevention and control of dengue depends on effective vector control measures. Use of acetylsalicylic acid, non-steroidal anti-inflammatory drugs and other drugs with effect on blood platelets and clotting factors is not recommended. Prophylactic platelet transfusions are given in dengue fever with thrombocytopenia to prevent hemorrhagic complications. Although the use of prophylactic platelet transfusions is increasing in countries where dengue is endemic, it is associated with risks and has financial implications. ${ }^{10}$

Dengvaxia, a vaccine to prevent dengue, manufactured by Sanofi Pasteur, is approved and registered in severala countries for specific age group and is recommended only to be given to persons with confirmed prior dengue virus infection. In 2017, the manufacturer announced that the people who receive the vaccine and have not been previously infected with a dengue virus may be at risk of developing severe dengue if they get dengue after being vaccinated. ${ }^{11}$

\section{REFERENCES}

1. WHO. Surveillance-Dengue. (online article) Available from: https://www.who.int/westernpacific/ emergencies/surveillance/dengue. [Last accessed on $1^{\text {st }}$ May,2020].
2. National Health Portal. Government of India. (online article). Available from: https://www.nhp.gov.in/national-dengue-day pg

[Last accessed on $01^{\text {st }}$ May,2020].

3. WHO. Dengue and severe dengue. (online article) Available from: https://www.who.int/newsroom/fact-sheets/detail/dengue-and-severe-dengue [Last accessed on $1^{\text {st }}$ May,2020].

4. Sarkar JK, Chatterjee SN, Chakravarty SK. Haemorrhagic fever in Calcutta: some epidemiological observations. Indian J Med Res. 1964;52:651-9.

5. Chatterjee SN, Chakravarti SK, Mitra AC, Sarkar JK. Virological investigation of cases with neurological complications during the outbreak of haemorrhagic fever in Calcutta. J Indian Med Assoc. 1965;45:314-6. 6. Carey DE, Myers RM, Reuben R, Rodrigues FM. Studies on dengue in Vellore, South India. Am J Trop Med Hyg. 1966;15:580-7.

7. National Vector Borne Disease Control Programme. Government of India. Dengue/DHF sitation in India. (Online fact sheet). Available from: https://nvbdcp.gov.in/index4.php?lang=1Elevel=oElin

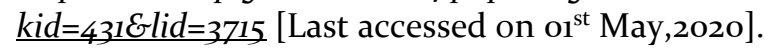

8. European Centre for Disease Prevention and Control. Aedes aegypti - Factsheet for experts (Online fact sheet). Available From: https://www.ecdc.europa.eu/en/disease-vectors/facts Lmosquito-factsheets/aedes-aegypti [Last accessed on $\mathrm{or}^{\text {st }}$ May,2020].

9.Reinhold JM, Lazzari CR, Lahondère C. Effects of the Environmental Temperature on Aedes aegypti and Aedes albopictus Mosquitoes: A Review. Insects. 2018;9(4): 158.

10. Kaur P, Kaur G. Transfusion support in patients with dengue fever. Int J Appl Basic Med Res. 2014; 4(Suppl 1): S8-S12.

11. Centers for Disease Control and Prevention (CDC). Dengue Vaccine. (Online Article). Available from: https://www.cdc.gov/dengue/prevention/denguevaccine.html [Last accessed on $01^{\text {st }}$ May,2020]. 